\title{
Sleeping Mode MODLEACH Protocol for WSN
}

\author{
Shiv Ashish Dhondiyal ${ }^{1}$, Deeepak Singh Rana ${ }^{2}$ \\ Asst. Professor, Department of Computer Science and Engineering, \\ Graphic Era Hill University, Dehradun, Uttarakhand, India ${ }^{1,2}$
}

\begin{abstract}
The world of wireless sensing element networks is one among the rising and quick growing field within the scientific world. The importance of wireless sensing element networks is because of its huge potential for sensing the elements in the environment. Because of these devices work on battery power and will be placed in hostile environments so commutation between them becomes a difficult task. Thus optimizing the energy of these networks becomes vital. In this paper we tend to propose SM - modleach protocol that could be a extended version of modleach protocol. The activity analysis of chosen protocol within the case of various scenario of affairs are fully reported in this paper. The analysis and experiments show that proposed protocol is economic and sensible and achieves higher performance than the previous approaches.
\end{abstract}

Keywords: WSN, SM-Modleach, Cluster head, cluster member

\section{INTRODUCTION}

WSNs sensors are deployed in an atmosphere to watch static or dynamic events. The activity of static events (such as temperature, wetness etc.) is extremely straightforward to hold out. On the opposite hand, dynamic events square measure usually non-cooperative event is that the movement of an unwanted vehicle in an exceedingly battle field and also the movement of whales within the ocean. They are dangerous to watch and that they aren't stable as they are going up and down. Therefore, it is extremely troublesome to check energy saving schemes for sensing of dynamic event. As an example a forest observation application involves static observation approach whereas a target chase application involves a dynamic observation approach. To optimize node's life time, we would like to specialize in such algorithms, protocols and physical circuitries which will create most out of restricted power supply. Therefore, this can be the foremost concern for scientists and researchers. Any technology that is in the method of its development provides a heap of challenges within the same approach, wireless sensing element networks do. Sensing, computing and transcribing by small sized sensors with power constraint is not an easy issue. In any network particularly wireless multi hop networks, for economic performance, its protocols should be terribly economical. Varied protocols square measure developed that address power downside in sensing element networks. Most outstanding routing algorithms is categorized into 3 sorts i.e. transmission mechanism algorithms; hop to hop transmission algorithms and cluster based mostly algorithms. Another downside that persists is to handle bulk of data perceived and missed out by each node of a network. (A WSN might incorporate thousands of nodes). For that information aggregation and information fusion algorithms work, but there are invariably areas for betterment. In an economical wireless sensing element network, we would like economical routing protocol that has low routing overhead and well organized information aggregation mechanisms to extend smart place of network and to save lots of restricted power of sensing element node. Sensor network needs bound protocol for economic performance. For example, protocols are available sort of a particular application with an outlined order to mixture information and optimizing energy consumption. This sort of protocol is observed as class-conscious routing.

\section{RELATED WORK}

Anar A.hady et al. [1] propose a Low-Energy Adaptive Clustering Hierarchy Centralized Sleeping Protocol (LEACHCS) for wireless sensor networks, and compare the results of proposed protocol with LEACH protocol.

D.Mehmood et.al. [2] has given a MODLEACH protocol by introducing economical cluster head replacement theme and twin transmission power levels.

Debabrata Singh et al. [3] proposed protocol Enhanced Modified LEACH (EMODLEACH) which is another reactive protocol which is implemented for homogeneous network model.

Heinzelman, et al. [4] purposed another (LEACH) based protocol that could be a cluster- based mostly protocol. A member node within the cluster should sense the forward and backward region to the atmosphere and transmit the perceived information to a cluster head.

A.Koucheryavy, et al. [5] the authors proposed a clustering routing protocol by the name of Enhanced LEACH, which extended LEACH protocol by balancing energy consumption in the network. 
Baiping Lil et al. [6], gives a low energy-consumption chain-based routing protocol LEACH-CC. The new protocol is characterized by each node will send information about its current location and energy level to BS.

Zahoor A.Khan et al. [7] authors have introduced an energy efficient routing protocol for wireless sensor network.

A.Ahamad et al. [8] proposed Density Controlled Divide-and-Rule LEACH. This protocol selects optimal number of CHs on the bases of nodes' density such that uniform distribution of load on CHs is maintained throughout the network operation.

Monu Ranil et al. [9] solves the problem of non-uniform load distribution on CHs by removing away CHs. This paper deals with the routing protocols for distributed wireless sensor networks.

\section{PROPOSED WORK}

This section explains in details the practicality of enhanced sleep mode MODLEACH protocol that could be a changed version of MODLEACH protocol. While planning and simulating planned algorithmic rule following assumptions square measure adopted square measure as follows:

This protocol provides a Sleeping Method for sensors inside the cluster to enhanced the life of sensors and also the overall period of time of the network. When first spherical energy of cluster head is checked.

In this protocol the network analyze the information sent by nodes, betting on this information and clusters head energy to finish information causing in an exceedingly spherical or square measure switched to sleeping mode till ensuing spherical.

The steps follow during this theme square measure as follow:

1. Select a cluster head with a likelihood $q$ having highest energy and keeping the remaining of the nodes in sleeping mode. Within the next round existing cluster has not pay a lot of energy throughout it will stay cluster head.

2. Each sensing element node generates a variable quantity and compares it with minimum threshold. If variable quantity worth but less then minimum threshold, then it becomes cluster head for this round.

3.The rest sensing elements are appointing by the base station to their respective cluster heads by choosing the minimum distance between the sensing element and the $\mathrm{CH}$.

4. A time frame slot is reserved by base station for $\mathrm{CH}$ to its cluster members to begin out inflicting data.

5. Then cluster head $(\mathrm{CH})$ collect all the information and sends it to the bottom station.

6.The sink receives the first info packet among the clusters from $\mathrm{CH}$. The bottom station evaluates info of the first frame and compare it to a given threshold, if this energy is below than threshold(T(s)), this means that this energy is not enough for it to be vital in this round.

7. The user consistent with the perceived application outlines this threshold.

8. The bottom station decides which sensors to go sleeping mode within cluster.

9. These nodes and their CH's reciprocally transfer their energy information to the sink.

\subsection{ALGORITHM}

\section{Notations Used}

q: Likelihood that a sensing element node becomes cluster head.

Em: Remaining energy of sensing element node.

En: Most energy a sensing element node will have.

$\mathrm{CH}$ : Cluster head

BS: Base station

CM: Cluster member

The algorithmic rule is divided into two phases:

1. Setup part

2. Steady state part

\section{SETUP part (Cluster Head Formation)}

Step 1: Every sensing element node 'S' generates a variable quantity $T \min (0<\mathrm{Tmin}<1)$.

Step 2: Calculate minimum threshold $\mathrm{T}(\mathrm{s})$.

$\mathrm{T}(\mathrm{S})=\operatorname{Max}(\mathrm{q} /\{(1-\mathrm{q}) * \bmod 1 / \mathrm{q})\} *[\mathrm{En} / \mathrm{Em}]$ if $\mathrm{S}$ belongs to $\mathrm{G}$

$\mathrm{T}(\mathrm{s})=0$ otherwise

Step 3: if $(\mathrm{Tmin}<\mathrm{T}((\mathrm{s}))$

Then sensing element node $\mathrm{S}$ becomes cluster head $(\mathrm{CH})$ for this current round ' $r$ '.

Else Sensing element node ' $\mathrm{S}$ ' becomes cluster member(CM).

Step 4: All cluster heads(CHs) advertise message to non-cluster head sensing element node to hitch them. 
Step 5: Every sensing element node be a part of the $\mathrm{CH}$ that is at minimum distance from it and kind the cluster.

Step 6: For every next round $(\mathrm{r}+1)$

if $(\mathrm{Tmin}<\mathrm{T}(\mathrm{S}))$

Then sensing element node $\mathrm{S}$ is still stay cluster head.

else

Go to step 2 to pick out new cluster head.

Step 7: Finish of setup part.

\section{STEADY STATE part (Data Transmission Phase)}

Step 1: Cluster head $(\mathrm{CH})$ allocate TDMA time slot to cluster members (CMs).

Step 2: Each CMs sends the data to $\mathrm{CH}$.

Step 3: Each $\mathrm{CH}$ aggregate the data and send first packet to base station(BS).

Step 4: if (send_packet< threshold)

then send_flag $(\mathrm{CH}, \mathrm{CM})=0$;

else send_flag $(\mathrm{CH}, \mathrm{CM})=1$;

Step 5: if (flag $=1$ )

then continue the round and send remaining data to BS.

else Put the entire cluster into sleeping mode for this round.

Step 6 : End of data transmission phase.

Step 7: End of algorithm

\section{Simulation Results and Analysis}

The planned approach is simulated mistreatment MATLAB 2012a. The nodes square measure at random deployed among the world of $100 \mathrm{~m} \mathrm{X} 100 \mathrm{~m}$. The bottom station is found at the middle of the preparation space with coordinates $50 \mathrm{~m}$ X 50m. The population of nodes for this simulation is one hundred $(n=100)$. The different samples of randomly deployed nodes are used for each simulation and the results discussed in the next sections are the average values of 30 simulations.

Table 1: Parameters used in simulation

\begin{tabular}{|l|l|}
\hline Network Size & $100 \mathrm{X} 100 \mathrm{~m} 2$ \\
\hline Initial Energy of Sensor Nodes & $00.5 \mathrm{~J}$ \\
\hline Packet Size & $4000 \mathrm{bits}$ \\
\hline Transceiver idle state energy consumption & $50 \mathrm{~nJ} / \mathrm{bit}$ \\
\hline Data Aggregation/ Fusion Energy consumption & $5 \mathrm{~nJ} / \mathrm{bit} / \mathrm{report}$ \\
\hline Amplification Energy (Cluster to BS) d $>=\mathrm{do}$ & $\mathrm{Efs}=10 \mathrm{pJ} / \mathrm{bit} / \mathrm{m} 2$ \\
\hline Amplification Energy (Cluster to BS) $\mathrm{d}=<\mathrm{do}$ & $\mathrm{Emp}=0.0013 \mathrm{pJ} / \mathrm{bit} / \mathrm{m} 2$ \\
\hline Amplification Energy (Intra Cluster Comm.) d $>=\mathrm{d} 1$ & $\mathrm{Efs} / 10=\mathrm{Efs} 1$ \\
\hline Amplification Energy (Intra Cluster Comm.)d $=<\mathrm{d} 1$ & $\mathrm{Emp} / 10=\mathrm{Emp} 1$ \\
\hline
\end{tabular}

Network Lifetime: The quantity between the beginning of the network operation and also the death of the last sensing element node is named network period of time.

Number of Cluster Heads per Cluster: The full range of nodes hand-picked as cluster heads from the entire network in each round.

Number of Alive Nodes: The full range of sensing element nodes that haven't nonetheless depleted all of their energy.

Number of Dead Nodes: The full range of sensing element nodes that have consumed all of their energy and aren't able to do any quite practicality.

\section{SIMULATION RESULTS}

The full range of rounds used for our experiments is ten thousand. Simulations of SM-MODLEACH compared with LEACH and MODLEACH square measure performed to look at the frequency of dead and alive nodes per spherical and range of packets transmitted to base station per spherical. 
Vol. 7, Issue 8, August 2018

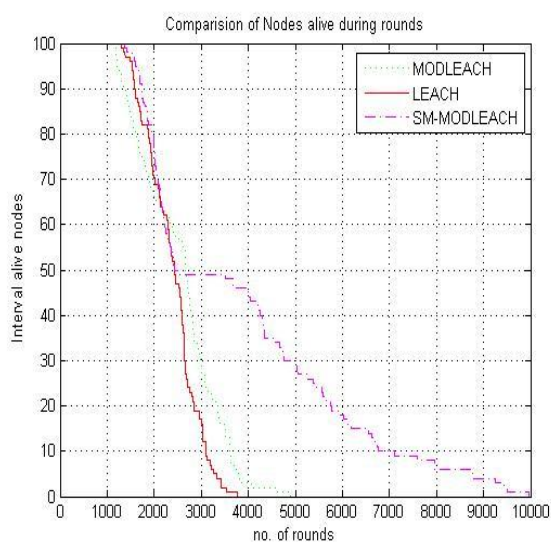

Figure 4.1: Total range of alive nodes in each round.

Figure 4.1 shows that SM-MODLEACH has an economical range of alive nodes owing to sleeping mode agglomeration. LEACH, MODLEACH choose \{the range the amount the quantity\} of cluster heads mistreatment distributed algorithms whereas a set number of cluster heads is chosen throughout every spherical in SM-MODLEACH conjointly energy of sensing element nodes square measure utilizes owing to sleeping mode conception.

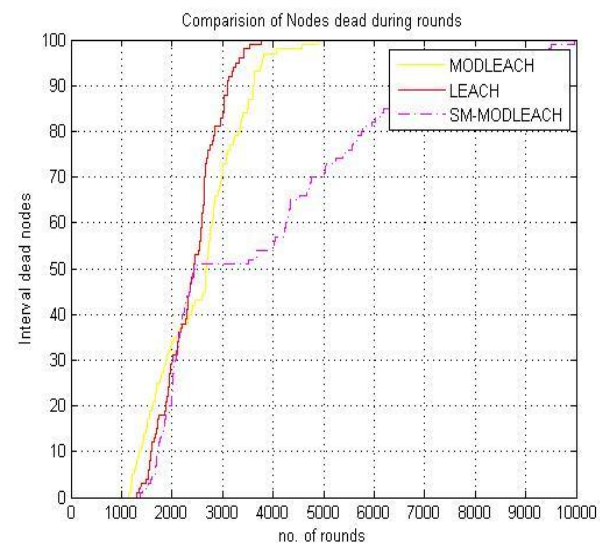

Figure 4.2: Total range of dead nodes in every spherical.

Figure 4.2 shows that SM-MODLEACH has bigger stability time as compared to LEACH and MODLEACH. the primary node of SM-MODLEACH is dead when close to 1400 rounds whereas the primary node of LEACH, and MODLEACH is dead when close to 1100 and 1200 (approx.) rounds severally.

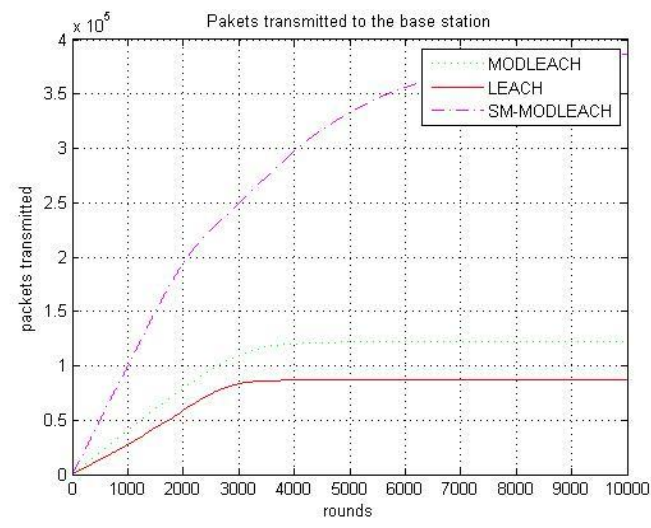

Figure 4.3: Packets transmitted to base station.

Figure 4.3 shows that range of packet transmitted to base station per round. It is terribly clear that enhance SMMODLEACH have less range of packets transmitted to the bottom station this can be owing to sleeping mode conception. 


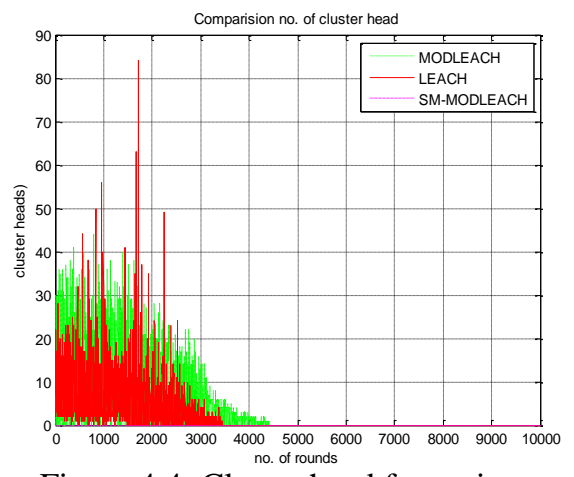

Figure 4.4: Cluster head formations

Figure 4.4 Shows that range of clusters fashioned by all 3 protocols, it's terribly clear that range of cluster head formation in SM-MODLEACH is extremely less as compared to LEACH and MODLEACH. Leach protocol have sizable amount of cluster head formation since in each spherical new cluster head is made. whereas sm-modleach have least range of cluster head formation as a result of sleeping mode conception.

\section{CONCLUSION AND FUTURE WORK}

In this paper, we tend to plan sleeping mode clustering (SM-MODLEACH) scheme for WSNs. The most focus was to reinforce network time period of MODLEACH protocol. In SM-MODLEACH, cluster heads square measure handpicked in every cluster on the idea of residual node energy and worth of perceived information whereas remainder of the clusters square measure sleeping. In our planned strategy, the soundness amount of network and network period of time are optimized. Simulation results show that there are important improvement altogether these parameters when put next with existing routing protocols LEACH and MODLEACH. Next improvement is probably by considering dependableness of knowledge and to confirm eminent delivery of knowledge, and conjointly style of a more robust routing protocol just in case once $\mathrm{CH}$ dies before sending the information to the $\mathrm{BS}$.

\section{REFERENCES}

[1]. Anar A.hady, Sherine M.Abd EI-Kader, Hussin S.Eissa.Intelligent Sleeping Mechanism For Wireless sensor network", Egyptian Informatics journal,2013.

[2]. D. Mahmood, N. Javaid, S. Mehmood, S. Qureshi, A.M. Memon, T. Zaman, "MODLEACH: a variant of LEACH for WSNs twenty sixth IEEE Canadian Conference on Electrical and pc Engineering (CCECE 2013), Regina, Canadian province, Canada, 2013.

[3]. Debabrata Singh, Sanjeet Kumar Nayak. "Enhanced modified Leach (EMODLEACH) protocol for WSN",2015 International Symposium on Advanced Computing and Communication(ISACC), 2015

[4]. W. Heinzelman, A. Chandrakasan, and H. Balakrishnan. "Energy-Efficient Communication Protocols for Wireless Microsensor Networks".In Proceedings of Hawaiian International Conference on Systems Science, Gregorian calendar month 2000.

[5]. A.Koucheryavy, Ahmed Carlos, and WalidOsamy, "Enhanced LEACH Protocol for Wireless sensing element Networks", St. Petersburg University of Telecommunication, 2009.

[6]. Baiping Li1, a, Xiaoqin Zhang, "Research and Improvement of LEACH Protocol for Wireless sensing element Network, International Conference on info Engineering, 2012.

[7]. Zahoor A.Khan, Srinivas Sampalli. AZR- LEACH: “An Energy Efficient Routing Protocol for Wireless sensor Network”, International Journal of Communication, Network and System Sciences.

[8]. A. Ahmad, K. Latif, N. Javaid, Z. A. Khan and U. Qasim, "density controlled divide-and rule theme for energy economical routing in wireless sensing element networks", 26th ieee.

[9]. N. N. Karnik and J. M. Mendel, “An introduction to type-2 mathematical logic systems,” Univ. Southern Golden State, 1. a., CA, USA, Tech. Rep., 1998.

[10]. S.Ahmed, M. M. Sandhu, N. Amjad, A. Haider, M. Akbar, A. Ahmad, Z. A. Khan*, U. Qasim\#, N. Javaid COMSATS Institute of data Technology, Islamabad, Pakistan. Internetworking Program, college of Engineering, Dalhousie University, Halifax, Canada. University of Canadian province, Alberta, Canada. iMOD LEACH: improved changed LEACH Protocol for Wireless sensing element Networks.

[11]. A. Iqbal, M. Akbar, N. Javaid, S.H. Bouk, M. Ilahi, R. D. Khan, "Advanced LEACH: A Static Clustering-based Heteroneous Routing Protocol for WSNs", J. Basic. Appl. Sci. Res., 3(5)864-872, 2013.

[12]. Congestion management in WSN mistreatment Cluster and adaptation Load Balanced Routing Protocol.

[13]. Energy economical theme for agglomeration Protocol Prolonging the period of time of Heterogeneous Wireless sensing element Networks Parul Saini, Ajay K Sharma Department of engineering \& Engineering National Institute of Technology Jalandhar, Punjab, India.

[14]. Fan Tibeto-Burman, Narayanan Sadagopan, Ahmed Helmy, "The vital framework for analyzing the Impact of quality on performance Of Routing Protocols for Adhoc Networks", Elsevier Journal of impromptu Networks, 2003, pp. 383-403.

[15]. Geng, Feng, and Shengjun Xue. "A comparative study of quality models within the performance analysis of MCL." Wireless and Optical Communication Conference (WOCC), 2013 22nd. IEEE, 2013.

[16]. B. Liang and Z. Haas. "Predictive distance-based quality management for PCS networks", In Proceedings of the Joint Conference of the IEEE pc and Communications Societies (INFOCOM), March 1999.

[17]. P. Nayak and D. Anurag, "A fuzzy logic-based agglomeration algorithmic rule for WSN to increase the network period of time," IEEE sensing element J., vol. 16, no. 1, pp. 137-144, Jan. 2016.

[18]. M. Misadeghi, A. Mahani, and M. Shojaee, “A novel distributed clustering protocol using fuzzy logic,” Procedia Technol., vol. 17, pp. 742748, Jan. 2014. 